\title{
Choledochoduodenostomy as an Alternative to Choledochojejunostomy for Biliary Reconstruction in Liver Transplantation
}

\author{
Qifei Wu • Chang Liu · Jianhui Li · Xiaogang Zhang • \\ Liang Yu $\cdot$ Yi Lv
}

Published online: 5 February 2010

(C) Société Internationale de Chirurgie 2010

We read with interest the article by Bennet et al., who advocated the use of choledochoduodenostomy (CDD) as an alternative method of achieving biliary drainage of the transplanted liver [1]. Although Wigmore had written a commentary for this article [2], we still have the following concerns.

First, the criteria for entry in this study were not clear, particularly concerning the selection for those patients who underwent $\mathrm{CDD}$ or choledochoenterostomy. From the context of the article, the subjects for this study were not selected for CDD or choledochoenterostomy randomly. We think the selected criteria may influence the effects of the operations. The authors noted that no patient during followup had any anastomotic strictures or significant reflux and subsequent cholangitis. Were there any symptoms or signs suggesting partial reflux or stricture of the anastomosis during the postoperative period? Did the authors provide some interference?

Second, a previous study showed that the postoperative recurrent cholangitis caused by CDD was observed mainly in patients with a narrow common bile duct (CBD) [3]. However, most CBDs in liver donors are in physiological condition, the diameters of which are less than those stated in the criteria. In their article, Bennett et al. noted that no patient during follow-up had subsequent cholangitis after the CDD. Just as the authors declaimed their brief followup may be a reason, we want to know whether they made some modification in the operative procedure that might lower the incidence of cholangitis.

Third, we discovered that the line drawings of the CDD may be not precise. The remaining biliary tract of the donor was too long, which may cause necrosis of the proximal biliary tract due to a limited blood supply. Although these problems are minor, they may cause misconceptions by the readers.

\section{References}

1. Bennet W, Zimmerman MA, Campsen J et al (2009) Choledochoduodenostomy is a safe alternative to Roux-en-Y choledochojejunostomy for biliary reconstruction in liver transplantation. World J Surg 33:1022-1025

2. Wigmore SJ (2009) Choledochoduodenostomy as an alternative to choledochojejunostomy in liver transplantation. World J Surg 33:1026-1027

3. Aldrete JS (2000) Choledochoduodenostomy. Oper Tech Gen Surg 2:304-310

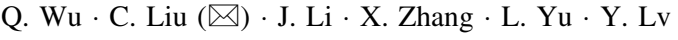
Department of Hepatobiliary Surgery, First Affiliated Hospital, School of Medicine, Xi' an Jiaotong University, Yan Ta West Road No. 277, Xi'an, Shannxi Province 710061, China e-mail: liuchangdoctor@163.com 